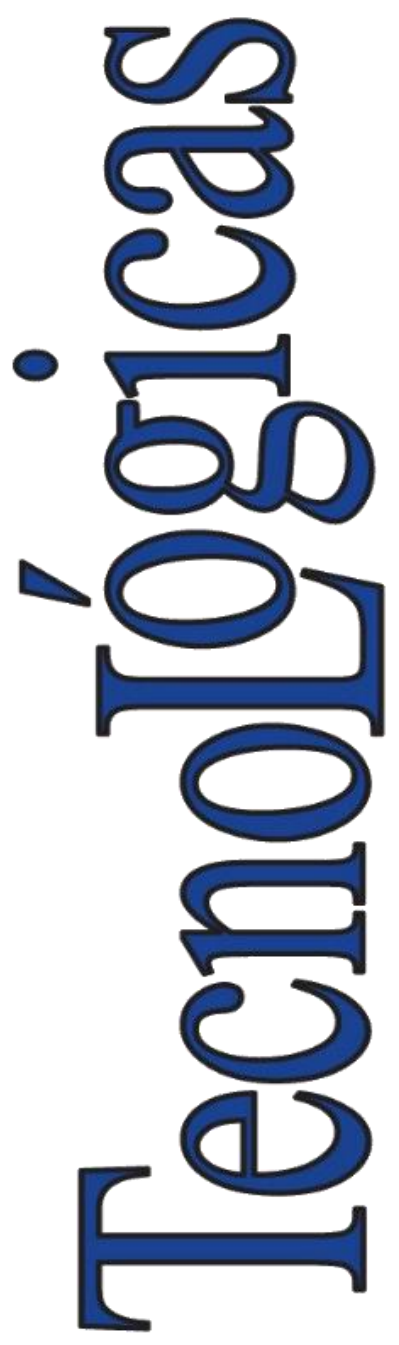

ISSN-p: 0123-7799 ISSN-e: $2256-5337$

Vol. 24, nro. 50, e1580

Recibido: 21 enero 2020 Aceptado: 10 agosto 2020 Disponible: 10 noviembre 2020

CInstituto Tecnológico Metropolitano Este trabajo está licenciado bajo una Licencia Internacional Creative Commons Atribución (CC BY-NC-SA)

\section{Estimación y modelización de la dispersión de black carbon en el Valle de Aburrá, Colombia}

\section{Estimation and Modeling of Black Carbon in Aburrá Valley, Colombia}

\author{
DJuan Camilo Roldán-Vargas ${ }^{1}$; \\ (iD) OMaría Victoria Toro-Gómez²; \\ iD Alejandro Marín-Sánchez ${ }^{3}$
}

${ }^{1}$ Universidad Pontificia Bolivariana, Medellín-Colombia, croldan20@hotmail.com ${ }^{2}$ Universidad Pontificia Bolivariana, Medellín-Colombia, victoria.toro@upb.edu.co

${ }^{3}$ Universidad Pontificia Bolivariana, Medellín-Colombia, alejandro.marinsa@upb.edu.co

Cómo citar / How to cite

J. C. Roldán-Vargas; M. V. Toro-Gómez; A. Marín-Sánchez, "Estimación y modelización de la dispersión de black carbón en el Valle de Aburrá, Colombia”, TecnoLógicas, vol. 23, nro. 50, e1580, 2021.

https://doi.org/10.22430/22565337.1580 


\title{
Resumen
}

A partir del inventario de emisiones del Valle de Aburrá (año 2015), ubicado en el departamento de Antioquia - Colombia, en este estudio se presenta la estimación de emisiones de Black Carbón (BC) de la región, así como la dispersión de este contaminante y la evaluación del Modelo de Calidad del Aire Comprehensivo con Extensiones (CAMx) y el modelo BRAMS, en la zona de estudio. Las emisiones totales estimadas de BC fueron de 850 toneladas donde el $97 \%$ de éstas se deben al sector transporte, donde la categoría camiones seguido de la categoría volquetas son los mayores emisores de este contaminante, aportando el $43 \%$ y $37 \%$, respectivamente. El combustible diésel es el principal responsable por las emisiones de BC con el $95 \%$ del total de las emisiones. La concentración máxima de BC para el 9 de noviembre de 2016 en el punto estudiado, fue de $8.47 \mu \mathrm{g} / \mathrm{m}^{3}$ a las 7 horas, donde el área de mayor impacto se encuentra localizada en el centro de Medellín y el norte de este municipio. El desempeño del modelo CAMx (inferencia difusa) para predecir las concentraciones de BC para el día de estudio, se encuentra en el $66 \%$. El modelo BRAMS se ajusta a los datos reales de las variables meteorológicas estudiadas (temperatura y humedad relativa), con errores MAPE menores al $3 \%$, para el día en que el modelo fue evaluado.

\section{Palabras clave}

Black Carbon, inventario de emisiones atmosféricas, CAMx, calidad del aire.

\begin{abstract}
Based on an inventory of atmospheric emissions in Aburrá Valley (Antioquia, Colombia) completed in 2015, this study presents an estimate of Black Carbon (BC) emissions in this region and the dispersion of this pollutant for November 9, 2016, as well as an evaluation of the CAMx and BRAMS models in the area under study. The estimated total emissions of BC were 850 tons, $97 \%$ of which were due to the transport sector. In the latter, trucks, followed by dump trucks, were the largest emitters of this pollutant, contributing $43 \%$ and $37 \%$, respectively. Diesel fuel was the main cause of $\mathrm{BC}$ emissions, with $95 \%$ of the total emissions. The maximum concentration of BC estimated for November 9, 2016, was $8.47 \mu \mathrm{g} / \mathrm{m}^{3}$ at 7:00 hours, and the area of greater impact was located downtown Medellín and north of the city. The performance of the CAMx model (fuzzy inference) in terms of the prediction of BC concentrations for the day under study was $66 \%$. The BRAMS model fit the real data of the meteorological variables investigated here (temperature and relative humidity), with MAPE errors under $3 \%$ for the day on which the model was evaluated.
\end{abstract}

\section{Keywords}

Black Carbon, atmospheric emission inventory, CAMx, air quality. 


\section{INTRODUCCIÓN}

Los aerosoles son partículas finas en estado sólido o líquido que se encuentran suspendidas en un gas; se originan de forma natural (biogénicos) o por actividades humanas (antropogénicos) y su rango de tamaño está en el orden de unos cuantos nanómetros hasta decenas de micras [1]. Un componente que puede dar origen a los aerosoles y que se emite a la atmósfera es el contaminante denominado Black Carbon (BC), que incluye componentes como Elemental Carbon (EC) y Organic Carbon (OC) [2], material carbonáceo producido por la combustión incompleta de biomasa, combustibles fósiles y biocombustibles [3],[4].

El $B C$ se caracteriza por absorber radiación en todas las longitudes de onda y se ha establecido que puede causar calentamiento de la atmósfera cuando se encuentra suspendido en el aire, y derretimiento de la nieve o el hielo tras su deposición [5]. Varios autores han estudiado la contribución del $B C$ al cambio climático, concluyendo que tiene un efecto neto de calentamiento, ocupando junto al $\mathrm{CO}_{2}$ y el metano, los primeros lugares entre los contaminantes que mayor aporte realizan al calentamiento global [5]-[6].

Los resultados acerca de la contribución del $B C$ al calentamiento global se basan en su mayoría en cálculos de forzamiento radiactivo, es decir del balance entre la radiación solar entrante y la infrarroja saliente. El Panel Intergubernamental sobre el Cambio Climático (2007) (IPCC por sus siglas en inglés) reportó tres tipos de forzamientos que al combinarse dan como resultado un forzamiento neto, dichos tipos son: Forzamiento directo, que se refiere a la absorción directa de la radiación solar o terrestre; forzamiento albedo nieve/hielo, resultado del cambio en la reflectividad de la nieve o el hielo a causa de su oscurecimiento por el $B C$; y forzamiento indirecto, resultante del impacto sobre las características de las nubes, como tiempo de vida, composición, reflectividad, etc.

El IPCC [5], Bond [7], entre otros, reportan valores diferentes para el forzamiento radiactivo ejercido por el $B C$, evidenciando que existe aún mucha incertidumbre sobre la magnitud del mismo [2].

Además de su impacto sobre el clima y los fenómenos meteorológicos, se ha encontrado que el $B C$ causa problemas en la salud de las personas, afectando principalmente el sistema respiratorio debido a que es capaz de alcanzar los alveolos pulmonares al ser inhalado [6],[8].

Existe también evidencia de que personas expuestas a emisiones provenientes de motores diésel, las cuales contienen $B C$, pueden desarrollar cáncer de pulmón [9].

De acuerdo con estudios realizados, se ha establecido que las emisiones de $B C$ en países desarrollados han tenido una importante disminución en los últimos años, en parte debido a mejores sistemas de control y combustibles más limpios; mientras que en países en vía de desarrollo de América Latina, Asia y África ha ocurrido lo contrario. Se espera que estas tendencias continúen en las próximas décadas [10]. En el caso particular del Área Metropolitana del Valle de Aburrá, más de dos tercios del total de emisiones de PM$_{2.5}$ (del cual forma parte el $B C$ ) provienen de fuentes móviles, siendo el empleo del combustible diésel el responsable del $80 \%$ de las emisiones de este contaminante [11].

En vista de la importancia que reviste el $B C$ tanto para la salud humana, como para el calentamiento global, y tomando en cuenta que se trata de un contaminante de corta vida, es decir, que sus efectos sobre el clima son casi inmediatos, así como los beneficios de su mitigación [7],[8], se hace necesario conocer sus principales fuentes, su comportamiento en la atmósfera y su tiempo de vida en la región del Área Metropolitana del Valle de Aburrá.

Con el fin de dar cumplimiento a estos propósitos, se decidió emplear el Modelo Integrado de Calidad del Aire con Extensiones (CAMx, por sus siglas en inglés). De acuerdo con [11], CAMx es un modelo fotoquímico de dispersión euleriano que posibilita la evaluación de la contaminación gaseosa y particulada en diferentes escalas. Es capaz de simular la emisión de 
contaminantes, su dispersión, reacciones químicas y remoción de la atmósfera en un sistema tridimensional de mallas anidadas, a través de la solución de la ecuación de continuidad para cada especie.

Para la simulación de resultados se aplicó el Sistema de Modelización Atmosférico Regional Brasilero (BRAMS) que simula la meteorología, y la actualización del inventario de emisiones para el Valle de Aburrá año 2015, elaborado por la Universidad Pontifica Bolivariana (U.P.B.) y el Área Metropolitana del Valle de Aburrá (autoridad ambiental de la región).

En el presente artículo se presenta la estimación de emisiones de $B C$ en el Área Metropolitana del Valle de Aburrá a partir del inventario de emisiones de esta región (año 2015), la dispersión de este contaminante en la zona de estudio y la evaluación de los modelos (CAMx y BRAMS), comparando los resultados de simulación con los resultados medidos.

\section{METODOLOGíA}

\subsection{Configuración del modelo CAMx}

\subsubsection{Descripción del modelo}

El campo de dispersión de las partículas de $B C$ se simulan usando el Modelo Integrado de Calidad del Aire con Extensiones (CAMx) que dispone de un módulo de especiación de varias especies químicas, incluyendo las partículas finas, específicamente la denominada Primary Elemental Carbon (PEC).

El modelo está diseñado para reunir todas las características técnicas especificadas en el estado del arte de los modelos de calidad del aire, todo en un sistema simple open-source (libre) que es computacionalmente eficiente, flexible y que está disponible públicamente [12].

Adicionalmente, se empleó el mecanismo de reacción químico Carbon Bond 6 (CB06) el cual describe la química oxidante troposférica de una manera concisa y adecuada para el uso en modelos atmosféricos complejos de tres dimensiones y el esquema de aerosol CF (Coarse/Fine) para representar la distribución de tamaño de partícula.

El modelo CAMx fue ejecutado con un dominio de resolución horizontal de 60 x $60 \mathrm{~km}^{2}$ y 13 niveles verticales. Este dominio cubre la región del Valle de Aburrá y parte del oriente en el departamento de Antioquia, tal y como se muestra en la Figura 1.

\subsection{2 Área de estudio}

El área de estudio se encuentra ubicada en el departamento de Antioquia, al noroccidente de Colombia y abarca la región del Área Metropolitana del Valle de Aburrá con sus diez municipios (Caldas, La Estrella, Sabaneta, Envigado, Itagüí, Medellín, Bello, Copacabana, Girardota y Barbosa), así como algunos municipios de la región del Oriente Antioqueño (Figura 1).

Es un valle con una altura promedio sobre el nivel del mar de 1480 metros, rodeado por montañas que alcanzan los $2800 \mathrm{msnm}$ y una topografía pendiente e irregular. Además, los municipios que conforman la región pertenecen a la cuenca hidrográfica del río Aburrá o Medellín, que atraviesa el valle de sur a norte. 

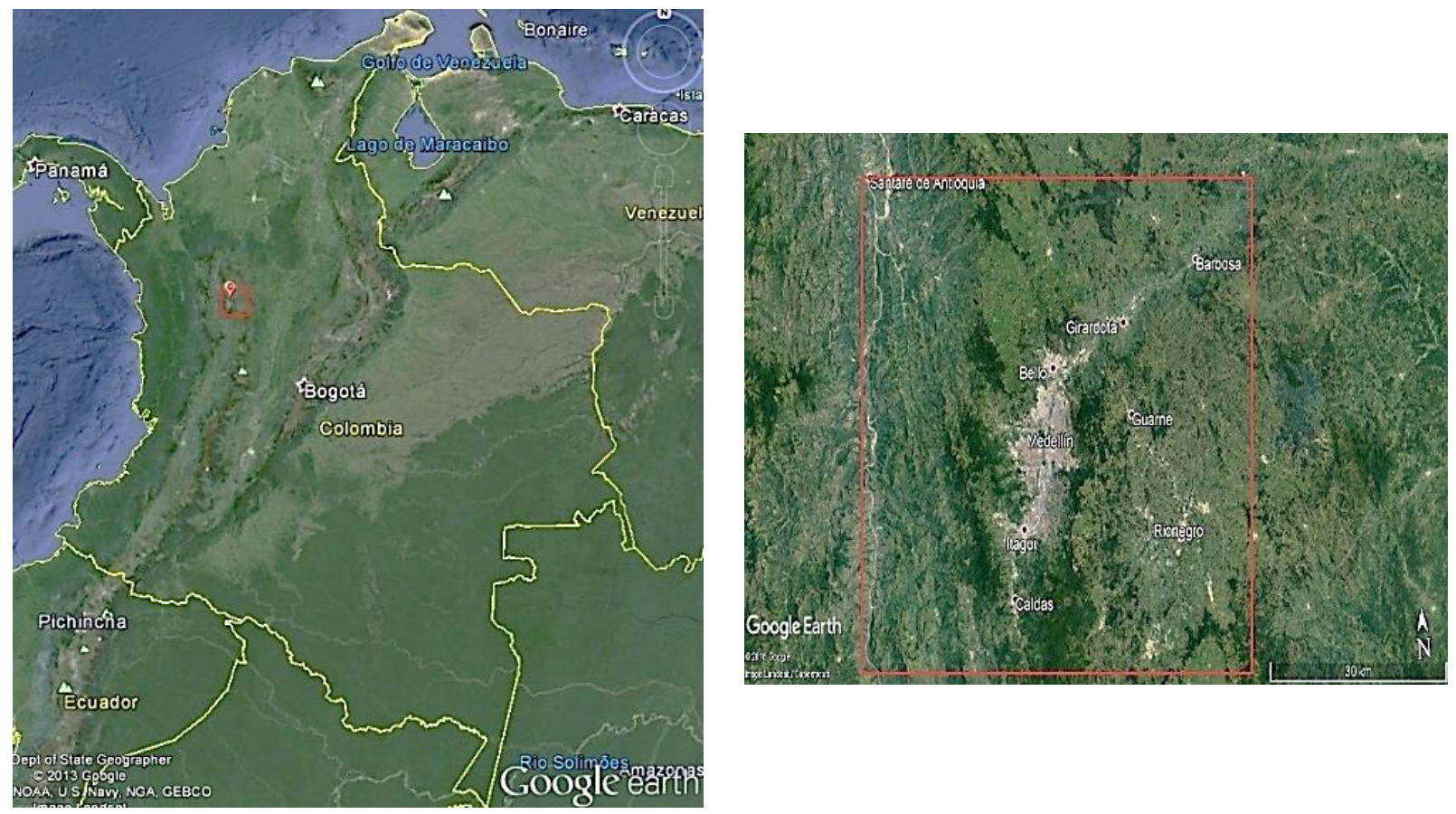

Figura 1. Dominio modelo CAMx. Fuente: Google Earth.

\subsubsection{Condiciones iniciales}

Las condiciones iniciales para el modelo fueron las sugeridas por Christopher Emery de ENVIRON [12] y se presentan en la Tabla 1.

Los parámetros meteorológicos alimentados al modelo se obtienen del Sistema de Modelización Atmosférico Regional Brasilero (BRAMS) versión 5, simulando la circulación atmosférica sobre un área geográfica limitada [13].

\subsubsection{Emisiones de fuentes contaminantes}

El Área Metropolitana del Valle de Aburrá, viene desarrollando desde el año 2000 el inventario de emisiones en convenio con la Universidad Pontificia Bolivariana. En el presente estudio se utiliza la última versión reportada, la cual es con año base 2015 [11] y se usa como datos de entrada al modelo fotoquímico CAMx.

Tabla 1. Condiciones iniciales para el modelo CAMx. Fuente: elaboración propia.

\begin{tabular}{cc}
\hline Compuesto & Valor \\
\hline NO & 0.00010000 \\
NO2 & 0.00100000 \\
O3 & 0.02000000 \\
OLE & 0.00000000 \\
PAR & 0.00307800 \\
TOL & 0.00000000 \\
XYL & 0.00000000 \\
HCHO & 0.00106800 \\
ALD2 & 0.00010510 \\
\hline
\end{tabular}

\begin{tabular}{cc}
\hline Compuesto & Valor \\
\hline ETH & 0.00000532 \\
PAN & 0.00003834 \\
$\mathrm{CO}$ & 0.10000000 \\
$\mathrm{HNO} 2$ & 0.00000073 \\
$\mathrm{H} 2 \mathrm{O} 2$ & 0.00226300 \\
$\mathrm{HNO} 3$ & 0.00152500 \\
ISOP & 0.00050000 \\
NXOY & 0.00000633 \\
\hline
\end{tabular}


El inventario tiene en cuenta las emisiones de fuentes de área, fijas y móviles de la región, que son estimadas a partir de factores de emisión (FE) reportados en el AP-42 Compilation of Air Pollution Emission Factor de la Agencia de Protección Ambiental de Estados Unidos (EPA) [11] para las dos primeras fuentes de emisión, mientras que para las móviles, se utilizan los FE reportados en el modelo Internacional de Emisiones Vehiculares (IVE por sus siglas en inglés) [14].

\subsection{Mecanismo de reacción}

El mecanismo de reacciones químicas que usa el modelo es el Carbon Bond (CB) que describe la química oxidante troposférica de una manera concisa y adecuada para el uso en modelos atmosféricos complejos en tres dimensiones. Las versiones existentes (CB4 y CB05) han sido usadas en modelos fotoquímicos como CMAQ, CAMx y WRF-Chem. En este estudio, se empleó el mecanismo CB6, con el cual se mejora la formación de oxidantes con la inclusión de varios compuestos orgánicos que son de larga duración y relativamente abundantes como propano, acetona, benceno y etino (acetileno). Adicionalmente, se mejora el modelizado de Aerosoles Orgánicos Secundarios (SOA) con la inclusión de compuestos alfa-dicarbonilo (glioxal y análogos) y la formación de peróxido de hidrógeno (H2O2) y, por lo tanto, la formación de aerosoles de sulfato con la actualización en la química de los radicales peroxi [12].

Las 213 sustancias químicas con las que cuenta el inventario de emisiones del Valle de Aburrá son agrupadas por especies funcionales que producen 39 ecuaciones de especies reactantes, las cuales hacen finalmente el mecanismo de reacción del modelo CAMx.

Además de utilizar dicho mecanismo de reacción, se cuenta con la herramienta para la especiación de partículas finas obtenida de la documentación de desarrollo de bases de datos de especiación de la EPA - Speciate Version 4.2 (EPA), donde se presentan las distribuciones (porcentaje en peso) de especies químicas para material particulado.

\subsection{Escenario modelizado}

Se realizó una simulación empleando el modelo CAMx para conocer la dispersión de BC en el Valle de Aburrá empleando el inventario para el día 9 de noviembre de 2016, el cual tiene condiciones meteorológicas favorables (verano) para una adecuada dispersión de contaminantes con alta radiación y baja nubosidad a baja altura.

El día mencionado fue evaluado en el punto donde se encuentra la estación automática de monitoreo de la calidad del aire ubicada dentro de la Universidad Pontificia Bolivariana $\left(75^{\circ} 35^{\prime} 27,08^{\prime \prime} \mathrm{O}, 6^{\circ} 14^{\prime} 36,85^{\prime \prime} \mathrm{N}\right)$, debido a que es una zona poblacional expuesta en donde es posible de evaluar el desempeño del modelo pues se cuenta con una campaña de medición del contaminante estudiado.

\subsection{Evaluación del escenario}

El Grupo de Higiene y Gestión Ambiental (GHYGAM) perteneciente al Politécnico Colombiano Jaime Isaza Cadavid, en convenio con el Área Metropolitana del Valle de Aburrá, realizó una campaña de medición de $\mathrm{BC}$ en la zona Laureles, específicamente en la Universidad Pontificia Bolivariana, durante 11 días del mes de noviembre de 2016 (del 2 al 5 , del 9 al 12 y del 16 al 18).

El monitoreo se llevó a cabo usando un medidor Automático de BC conocido en el mercado como Etalómetro Portable Magee Scientific Modelo AE42, el cual reporta en tiempo real a 
partir de la cuantificación de la tasa de cambio en la absorción de la luz transmitida, debido a la continua recolección del filtro de la deposición de aerosol. En el rango $880 \mathrm{~nm}$ es evaluada como medición directa de la concentración de BC pues representa la materia carbonácea tipo antracita que absorbe energía calórica [11]. Los datos recolectados en la campaña fueron usados para evaluar el desempeño del modelo CAMx.

En cuanto al análisis de desempeño del modelo meteorológico, se emplearon los datos de temperatura y humedad relativa proporcionados por la estación automática de monitoreo de la calidad del aire ubicada dentro de la Universidad Pontificia Bolivariana, zona Laureles, la cual, además, mide contaminantes como $\mathrm{SOx}, \mathrm{NOx}, \mathrm{O}_{3}$ y $\mathrm{PM}_{2.5}$.

\section{RESULTADOS Y DISCUSIÓN}

Una vez configurado el dominio computacional del modelo que contiene las variables topográficas y la definición del mecanismo de reacción química, se alimenta al modelo con las emisiones de $\mathrm{BC}$ con una desagregación espacial de 1 x $1 \mathrm{~km}^{2}$ y una resolución temporal de una hora.

\subsection{Estimación de emisiones de $B C$}

A partir de las emisiones de $\mathrm{PM}_{2.5}$ calculadas para fuentes fijas y móviles, provenientes del inventario de emisiones del Valle de Aburrá año 2015, se estimaron las respectivas emisiones de $\mathrm{BC}$ para cada una de las fuentes contaminantes, tal como se discute a continuación.

\subsection{Fuentes móviles}

Para las emisiones de BC provenientes de las fuentes móviles, se utilizó la documentación de desarrollo de bases de datos de especiación de la EPA - Speciate Version 4.2 (EPA), donde se presentan las distribuciones (porcentaje en peso) de especies químicas para material particulado. Es así como, a partir de esta distribución y de la emisión de $\mathrm{PM}_{2.5}$ calculado, es posible estimar las emisiones de BC.

En la Tabla 2 se presentan las emisiones anuales de BC discriminadas por categoría vehicular. Se observa que la categoría camiones seguido de las volquetas son los mayores emisores aportando el $43 \%$ y $37 \%$, respectivamente, del total de las emisiones asociadas a fuentes móviles.

Tabla 2. Emisiones anuales de $B C$ por categoría vehicular. Fuente: elaboración propia.

\begin{tabular}{cc}
\hline Categoría & $B C$ (ton/año) \\
\hline Camiones & 353 \\
Volquetas & 302 \\
Buses & 88 \\
Autos & 32 \\
Motos 4T & 22 \\
Tractocamiones & 17 \\
Taxis & 9 \\
Motos 2T & 2 \\
Total & 826 \\
\hline
\end{tabular}


Las emisiones anuales por tipo de combustible se presentan en la Tabla 3, donde se observa que el diésel es el principal responsable por las emisiones de BC con un $95 \%$ del total de las emisiones asociadas a fuentes móviles, el cual, a pesar de su versatilidad y su eficiencia, históricamente se ha evidenciado que producen altas emisiones de NOx y material particulado. Adicionalmente, se observa que la gran mayoría de las emisiones de BC proviene de las categorías Camiones y Volquetas, que principalmente utilizan el combustible diésel.

Tabla 3. Emisiones anuales de $B C$ por tipo de combustible. Fuente: elaboración propia.

\begin{tabular}{cc}
\hline Combustible & $B C$ (ton/año) \\
\hline Diésel & 785 \\
Gasolina & 38 \\
GNV & 3 \\
Total & 826 \\
\hline
\end{tabular}

\subsection{Fuentes fijas}

Utilizando la clasificación establecida en la Tabla 4, se muestran los resultados en la Tabla 5 de las emisiones anuales de BC por actividad productiva asociada con las fuentes fijas propias de la región.

A partir de los resultados de emisiones obtenidos para las diferentes actividades industriales, se concluye que el sector Textil es el que más aporta en emisión de este contaminante, seguido de la industria maderera con un $59 \%$ y $30 \%$, respectivamente, del total de las emisiones de BC asociadas a fuentes fijas. Los demás sectores productivos aportan muy poco a la emisión de este contaminante por lo que no se incluyeron en la Tabla 5.

Tabla 4. Clasificación de sectores por actividad productiva. Fuente: elaboración propia.

\begin{tabular}{|c|c|}
\hline Sector & Descripción \\
\hline BAT & Bebidas, alimentos y tabaco (incluidos alimentos para animales). \\
\hline TXT & Textil y de confección (procesamiento y producción de textiles incluyendo procesos de teñido). \\
\hline CVL & Cerámicos y vítreos (ladrilleras, alfareras, tejares e industrias de cerámica). \\
\hline PAP & Papel, cartón, pulpa e impresión. \\
\hline PCE & $\begin{array}{l}\text { Plásticos, cauchos y empaques (incluidas reencauchadoras, fabricación y procesamiento de } \\
\text { llantas). }\end{array}$ \\
\hline MMC & $\begin{array}{l}\text { Metalmecánico (fundición y manejo de metales, hierro, metales no ferrosos, producción de } \\
\text { maquinaria eléctrica y no eléctrica). }\end{array}$ \\
\hline QMC & $\begin{array}{l}\text { Química (producción de compuestos químicos, producción de jabones y detergentes, pinturas y } \\
\text { resinas). }\end{array}$ \\
\hline CUR & Cueros, curtimbres y calzado. \\
\hline ASF & $\begin{array}{l}\text { Derivados del petróleo (producción y procesamiento de asfaltos y emulsiones asfálticas, } \\
\text { explotación y tratamiento de triturados). }\end{array}$ \\
\hline TER & $\begin{array}{l}\text { Terciario (incluye empresas del sector terciario, comercial y de servicios que por su actividad } \\
\text { posean calderas u hornos eléctricos, por ejemplo, hoteles, hospitales, cementerios, lavanderías y } \\
\text { otros). }\end{array}$ \\
\hline MAD & Aserríos, depósitos de maderas, e industrias que trabajan la madera. \\
\hline OTR & $\begin{array}{l}\text { Otras industrias (en este sector se agrupan las industrias que no se pueden clasificar en ninguna } \\
\text { de las categorías anteriores). }\end{array}$ \\
\hline
\end{tabular}


Tabla 5. Emisiones anuales de $B C$ por sector productivo. Fuente: elaboración propia.

\begin{tabular}{cc}
\hline Sector & $B C$ (ton/año) \\
\hline TXT & 14.1 \\
MAD & 7.1 \\
BAT & 0.9 \\
PAP & 0.5 \\
QMC & 0.5 \\
CUR & 0.5 \\
MMC & 0.2 \\
CVL & 0.1 \\
ASF & 0.1 \\
Total & 24.0 \\
\hline
\end{tabular}

En la Tabla 6 se muestran las emisiones anuales por municipio, en donde se observa que el municipio de Barbosa realiza los mayores aportes de BC seguido del municipio de Itagüí con un $32 \%$ y $27 \%$, respectivamente, del total de las emisiones en el Valle de Aburrá. Los demás municipios del Valle de Aburrá aportan muy poco a la emisión de este contaminante.

La razón por la que el sector textil y maderero son los que más aportan a la emisión del $\mathrm{BC}$, así como el municipio de Barbosa, se debe a la distribución de las fuentes por sector en cada municipio.

Tabla 6. Emisiones anuales de $B C$ por municipio. Fuente: elaboración propia.

\begin{tabular}{cc}
\hline Municipio & $B C$ (ton/año) \\
\hline Barbosa & 7.2 \\
Itagüí & 6.4 \\
Bello & 5.2 \\
Girardota & 3.0 \\
Medellín & 0.7 \\
Copacabana & 0.4 \\
Envigado & 0.3 \\
La Estrella & 0.3 \\
Caldas & 0.2 \\
Sabaneta & 0.2 \\
Total & 24.0 \\
\hline
\end{tabular}

Debido a que en el municipio de Barbosa se encuentran las 3 empresas más grandes del sector maderero, éstas aportarían la mayor carga de BC en el municipio.

Mientras que, para el caso del sector textil, si bien emite el doble al maderero, esta industria se encuentra asentada en los diferentes municipios del Valle de Aburrá, principalmente en el municipio de Itagüí, siendo éste el segundo municipio emisor más importante.

\subsection{Comparación inventario de $B C$}

En total, en la región del Área Metropolitana del Valle de Aburrá para el año 2015, se emitieron 850 toneladas de BC, de las cuales el $97 \%$ pertenecen a las fuentes móviles, especialmente los vehículos que usan diésel, como son los camiones y las volquetas. 
En la Tabla 7 se muestran las estimaciones de emisión anuales de BC por diferentes inventarios globales y regionales para el año 2000, los cuales muestran que la variación de estas emisiones es considerablemente amplia, donde los rangos a nivel global varían de 4'500.000 a 12'600.000 Ton/año, mientras que los rangos a nivel regional varían de 260.000 a 1'800.000 Ton/año [15].

Tabla 7. Comparación de estimaciones de emisión anual de BC por diferentes inventarios Fuente: elaboración propia.

\begin{tabular}{cc}
\hline Escala & $B C$ (Ton/año) \\
\hline Global & $4^{\prime} 800.000$ \\
China & $1^{\prime} 500.000$ \\
India & 800.000 \\
Estados Unidos & 260.000 \\
\hline
\end{tabular}

En la Tabla 8 se muestra la comparación de emisiones de BC por cada 10000 habitantes para el Valle de Aburrá y los países de China e India, en donde se muestra que China tiene la mayor emisión de BC, la cual es aproximadamente cinco veces la emisión del Valle de Aburrá por cada 10000 habitantes.

Esto se debe al uso desmesurado del carbón como fuente de energía a causa de la alta industrialización, el transporte y el alto desarrollo económico de ese país.

Tabla 8. Comparación de estimaciones de emisión anual de $B C$ por cada 10000 habitantes por diferentes inventarios. Fuente: elaboración propia.

\begin{tabular}{cc}
\hline Región & $B C($ Ton x 10000 hab/población $)$ \\
\hline China & 11.88 \\
India & 7.59 \\
Valle de Aburrá & 2.15 \\
\hline
\end{tabular}

\subsection{Dispersión de $B C$ en el Valle de Aburrá}

En la Figura 2 se muestra el perfil horario de las concentraciones de BC para el día 9 de noviembre de 2016, en donde se puede observar un pico máximo de concentración de $8.47 \mu \mathrm{g} / \mathrm{m}^{3}$ a las 7 horas. En laFigura 3 Figura 3, se muestra la dispersión de BC resultado de la modelización en CAMx, para el día de estudio a las 7 horas, donde, de acuerdo con los resultados, se presentan las mayores concentraciones. En la dispersión de BC se puede evidenciar que el área de mayor impacto se encuentra localizada en el centro de Medellín y el norte de este municipio, la cual sigue la dirección de viento en esta zona de estudio.

\subsection{Evaluación y verificación del modelo BRAMS}

La evaluación del desempeño del modelo se realizó con la comparación de las variables temperatura y humedad relativa del aire como parámetros de comparación entre los datos simulados y los medidos para el día 9 de noviembre de 2016. Posteriormente, se realizó la verificación del modelo comparando dichos parámetros para el día 16 de noviembre de 2016.

Los dos días son evaluados en el punto donde se encuentra la estación automática de monitoreo de la calidad del aire ubicada dentro de la Universidad Pontificia Bolivariana. La evaluación y verificación del modelo meteorológico mostró que este representó la variabilidad diaria de la temperatura y humedad relativa en esta zona del Valle de Aburrá. 


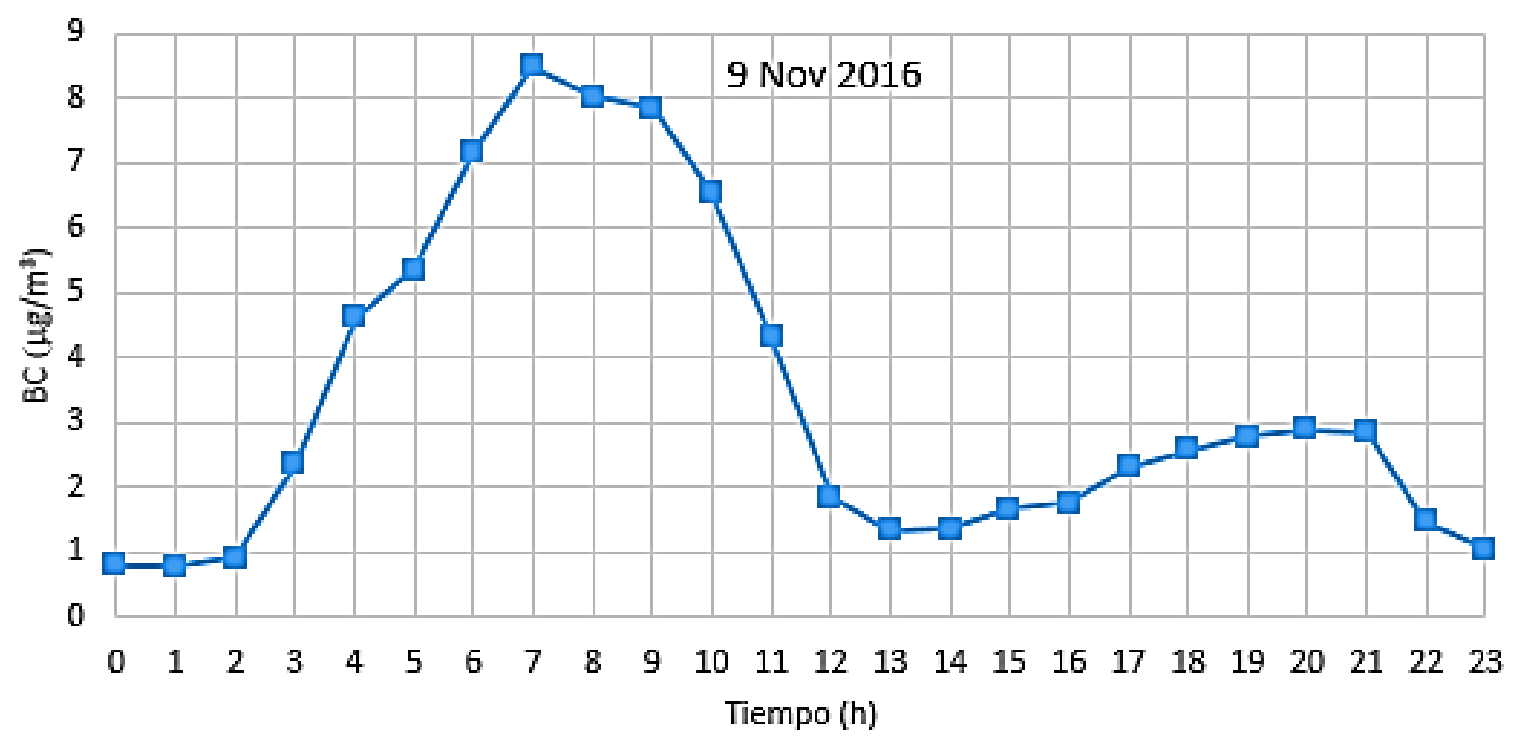

Figura 2. Perfil horario de las concentraciones de BC. Fuente: elaboración propia.

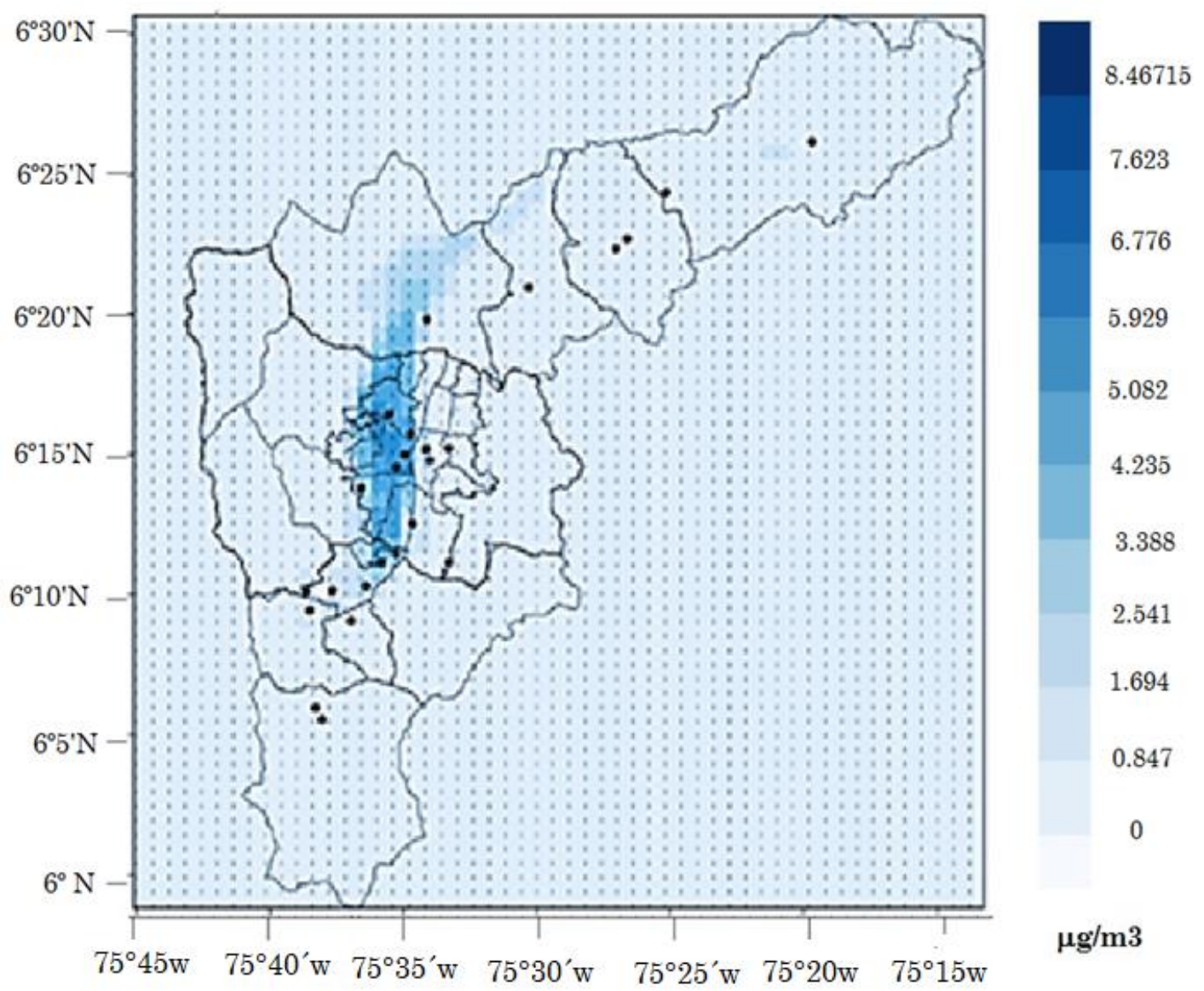

Figura 3. Dispersión de las concentraciones de BC. Fuente: elaboración propia.

Como se observa en la Figura 4, el modelo BRAMS se ajusta a los datos reales, mostrando una tendencia similar para el día en el que se evaluó el modelo y para el día en que se realizó la verificación del mismo. 


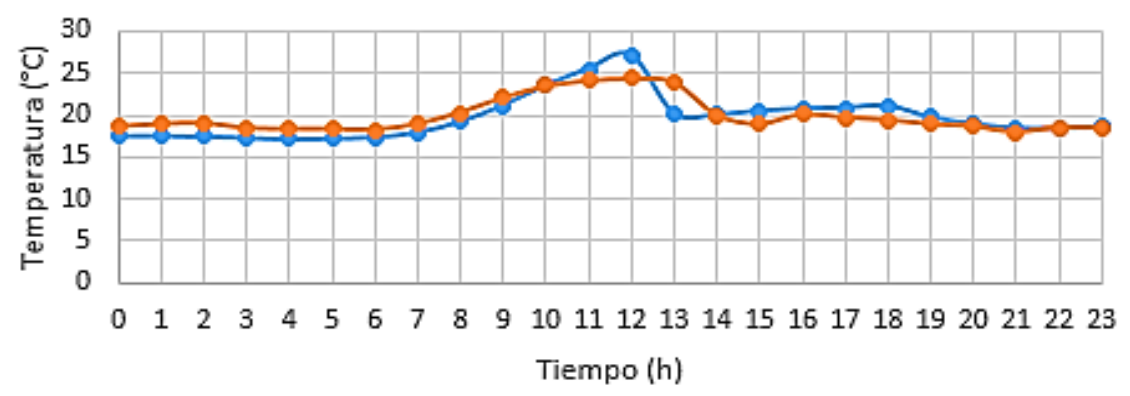

$\longrightarrow$-Simulado $\longrightarrow$ - Observado

Temperatura $\left({ }^{\circ} \mathrm{C}\right) 9$ de noviembre de 2016

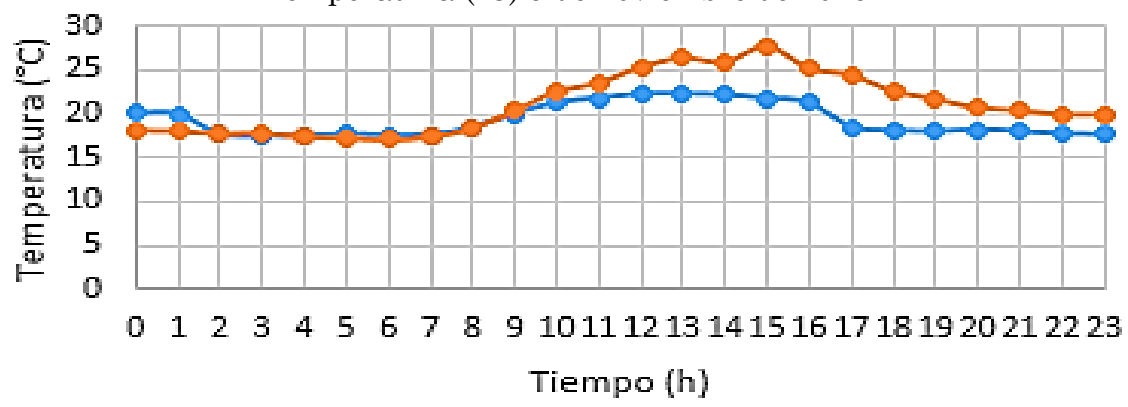

- Simulado —-Observado

Temperatura $\left({ }^{\circ} \mathrm{C}\right) 16$ de noviembre de 2016

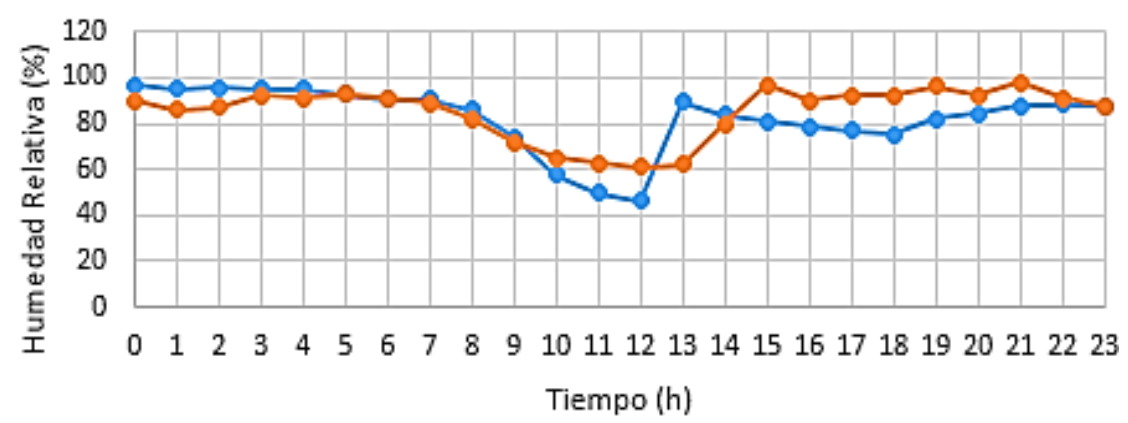

$\longrightarrow$ Simulado $\rightarrow-$ Observado

Humedad Rel. (\%) 9 de noviembre de 2016

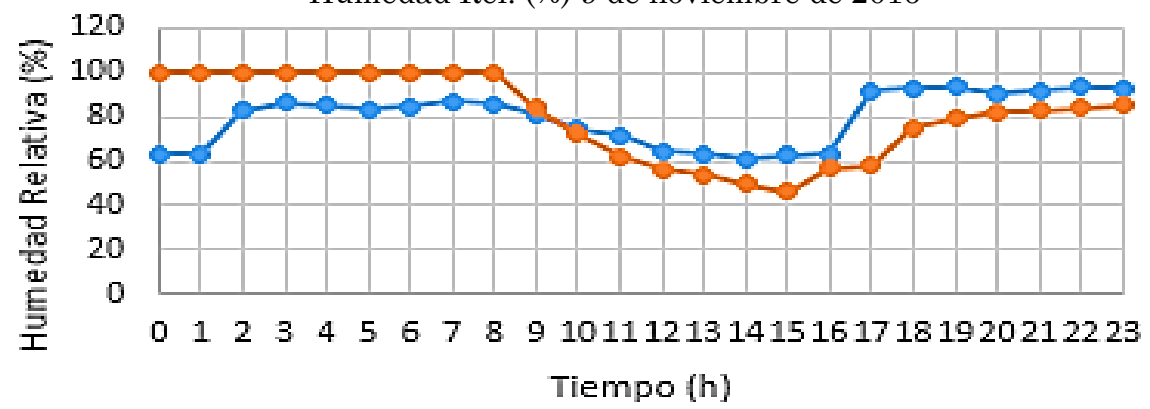

- Simulado - Observado

Humedad Rel. (\%) 16 de noviembre de 2016

Figura 4. Comparación del perfil horario de temperatura y humedad relativa, simulada y medida, para los días evaluados. Fuente: elaboración propia. 
El Error Porcentual Absoluto Medio (MAPE) de la temperatura y humedad relativa en los días evaluados, arrojó un valor de $0.8 \%$ y $2.6 \%$, respectivamente, para el 9 de noviembre y $7.7 \%$ y $3.4 \%$, respectivamente, para el 16 de noviembre de 2016, los cuales se consideran bajos (por debajo de $15 \%$ ) y, por lo tanto, de acuerdo con estos resultados, se corrobora que hay un buen ajuste del modelo BRAMS a las condiciones reales medidas en la estación automática de la Universidad Pontificia Bolivariana.

\subsection{Evaluación del modelo CAMx}

En primera instancia, en la Figura 5, se presenta un análisis cualitativo de la precisión del modelo CAMx al predecir las concentraciones horarias de BC para el 9 de noviembre de 2016. La Figura 5 indica que el modelo se encuentra sobre-estimando aproximadamente el 25 $\%$ de las concentraciones simuladas, mientras que el $67 \%$ de éstas se encontraron en la zona 1:1, es decir, los valores se ajustaron muy bien a la realidad. El $8 \%$ restante, muestra que el modelo subestimó las concentraciones, entregando valores menores a los medidos.

La inferencia difusa es una herramienta automática para la evaluación de los resultados simulados de calidad del aire [16], en la cual se obtiene un único indicador de confiabilidad o correlación (desempeño) para los datos, basándose en múltiples mediciones estadísticas indicadoras de confiabilidad/correlación.

El desempeño del modelo para predecir las concentraciones de BC en la estación automática U.P.B. se encuentra en el $66 \%$ para el día de estudio.

A continuación, se presenta la comparación de los perfiles horarios, simulado y observado, de las concentraciones de $\mathrm{BC}$ para el día de estudio, el cual tiene condiciones meteorológicas favorables para la dispersión de contaminantes y se encuentra dentro de la campaña de medición realizada por el grupo GHYGAM.

Como se muestra en la Figura 6, las concentraciones de BC simuladas se encuentran muy cerca de las medidas observando una tendencia muy similar en el perfil horario, subestimando ligeramente los datos en la mayoría del día analizado.

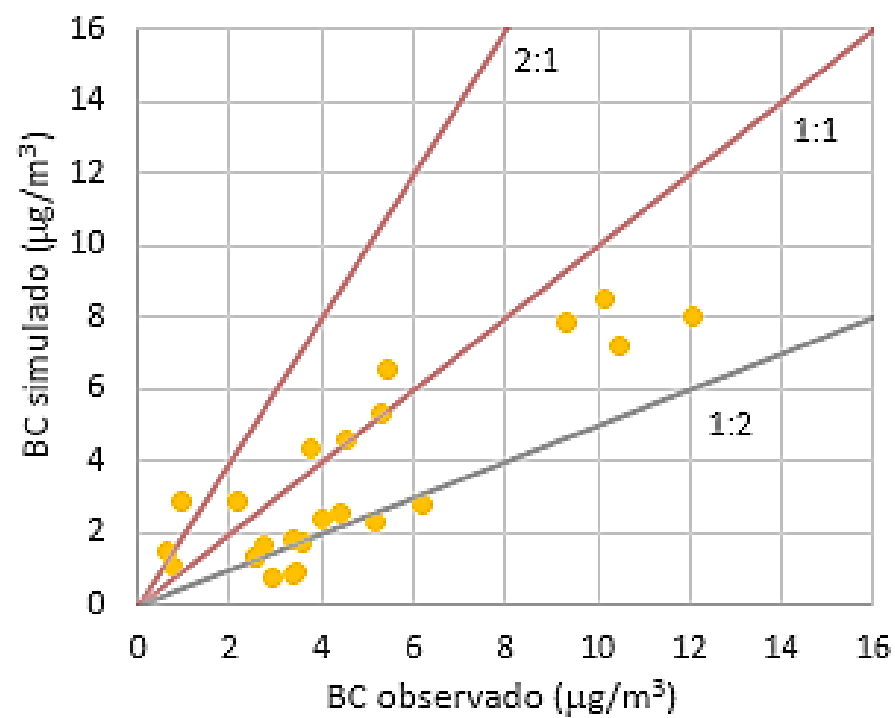

Figura 5. Comparación de la concentración de BC, simulada y medida, para el 9 de noviembre de 2016 Fuente: elaboración propia. 


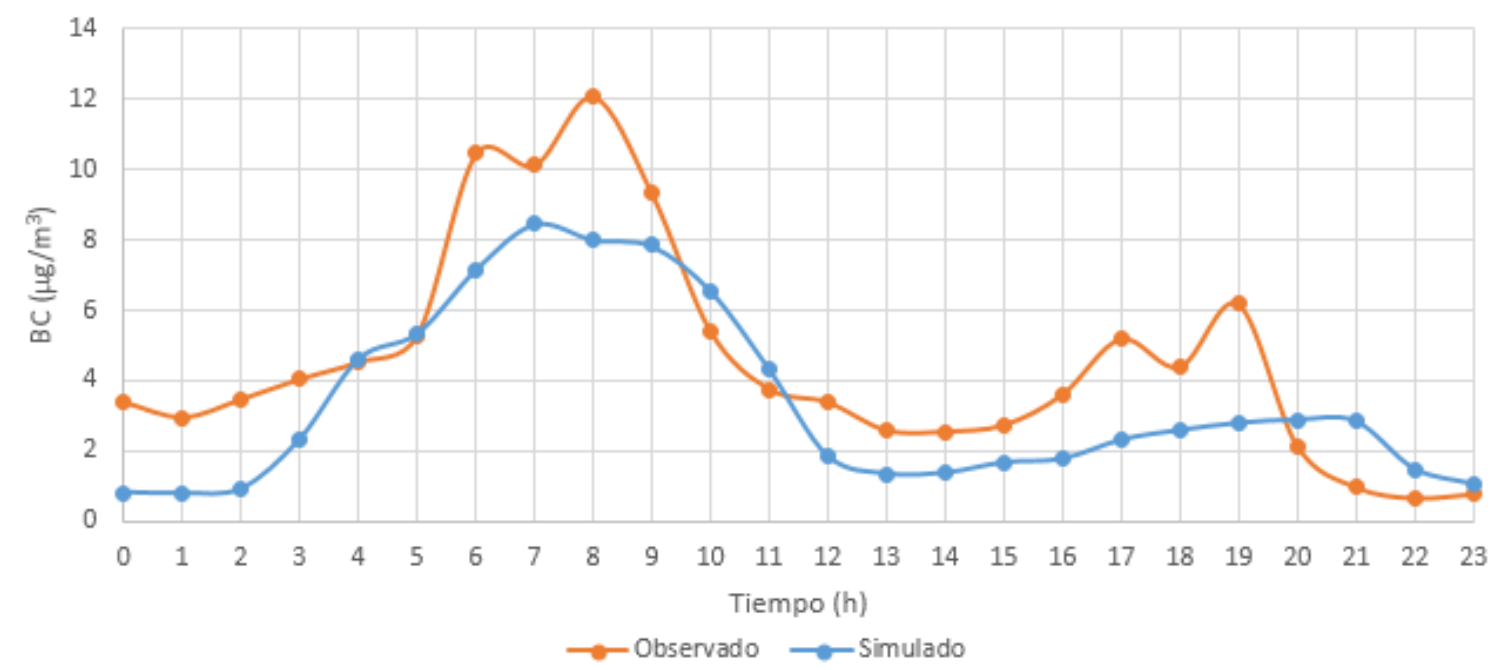

Figura 6. Comparación del perfil horario de la concentración de BC, simulada y medida, para el 9 de noviembre de 2016. Fuente: elaboración propia.

Adicionalmente, se observan dos máximos notables, uno en la mañana y otro en la tarde, pero de menor intensidad entre las 5 y las 10 horas y entre las 16 y las 19 horas, respectivamente, obteniendo un pico máximo de concentración $\mathrm{BC}$ observado de $12.09 \mu \mathrm{g} / \mathrm{m}^{3}$ mientras que el pico máximo de concentración BC simulada fue de $8.47 \mu \mathrm{g} / \mathrm{m}^{3}$. El Error Porcentual Absoluto Medio (MAPE) del día evaluado, arroja un valor de $12.7 \%$ por lo que se considera que hay un ajuste aceptable del modelo CAMx a las condiciones de BC medidas.

La eficiencia del modelo CAMx reportada en la literatura se ve influenciada de forma significativa por varios factores como el componente a modelizar, la meteorología y la locación, mostrando, en la mayoría de estudios, una subestimación con respecto a las concentraciones observadas [17].

En este estudio, las diferencias entre lo medido y lo observado en el modelo CAMx, se deben en gran parte a que en la malla de simulación no se encuentran aún representadas la totalidad de vías importantes en la zona de estudio (teniendo en cuenta que el $97 \%$ de las emisiones de BC se deben a las fuentes móviles), lo cual es una oportunidad de mejora para este Sistema de Modelización Atmosférica. Además, los errores en las suposiciones del modelo, su parametrización, inexactitud en los datos de entrada e incertidumbres relacionadas con la naturaleza estocástica de los procesos atmosféricos, son aspectos que se deben tener en cuenta para minimizar dichas diferencias.

\section{CONCLUSIONES}

A partir de la estimación de emisiones de BC para el Valle de Aburrá, se concluye que la categoría camiones seguido de la categoría volquetas son los mayores emisores del contaminante $\mathrm{BC}$ aportando el $41 \%$ y $38 \%$, respectivamente del total de las emisiones pertenecientes a fuentes móviles.

El combustible diésel es el principal responsable por las emisiones de BC seguido de la gasolina, con el $96 \%$ y $4 \%$, respectivamente.

El sector textil es el que más aporta en emisión de este contaminante seguido de la industria maderera, con un $59 \%$ y $30 \%$, respectivamente, del total de las emisiones de BC asociadas a fuentes fijas. 
El municipio de Barbosa realiza los mayores aportes de BC seguido del municipio de Itagüí, con un 30 \% y 27 \%, respectivamente, del total de las emisiones en el Valle de Aburrá pertenecientes a fuentes fijas.

Para el día de estudio (9 de noviembre de 2016), se obtuvo un comportamiento del perfil horario de las concentraciones de $\mathrm{BC}$ con un pico máximo de concentración para el día de verano de $8,47 \mu \mathrm{g} / \mathrm{m}^{3}$ a las 7 horas.

La dispersión de BC para el día de estudio, muestra que el área de mayor impacto se encuentra localizada en el centro de Medellín y el norte de este municipio.

El modelo meteorológico BRAMS se ajusta a los datos reales de las variables meteorológicas estudiadas (temperatura y humedad relativa), mostrando una tendencia similar para el día en el que se evaluó el modelo y para el día en que se realizó la verificación del mismo, con un error MAPE de la variable temperatura de $0.8 \%$ y $7.7 \%$, respectivamente, mientras que para la humedad relativa fue de $2.6 \%$ y $3.4 \%$, respectivamente.

El desempeño del modelo CAMx (inferencia difusa) para predecir las concentraciones de BC, en la estación automática U.P.B. para el día de estudio, se encuentra en el 66 \%. A su vez, el modelo se encuentra sobre-estimando aproximadamente el $25 \%$ de las concentraciones simuladas, mientras que el $67 \%$ de éstas se encontraron en la zona 1:1, es decir, los valores se ajustaron muy bien a la realidad. El 8 \% restante, muestra que el modelo subestimó las concentraciones entregando valores menores a los medidos.

El día 9 de noviembre de 2016, las concentraciones de BC simuladas se encuentran cerca de las medidas observando una tendencia muy similar en el perfil horario, subestimando ligeramente los datos en la mayoría del día analizado, en el cual el error MAPE es del $12.7 \%$, el cual es considerado bajo (por debajo de $15 \%$ ), por lo que se considera que hay un ajuste aceptable del modelo CAMx a las condiciones de BC medidas.

En este estudio, las diferencias entre lo medido y lo observado en el modelo CAMx se deben en gran parte a que en la malla de simulación no se encuentran aún representadas la totalidad de vías importantes en la zona de estudio (teniendo en cuenta que el $97 \%$ de las emisiones de $\mathrm{BC}$ se deben a las fuentes móviles), lo cual es una oportunidad de mejora para este Sistema de Modelización Atmosférica. Además, los errores en las suposiciones del modelo, su parametrización, inexactitud en los datos de entrada e incertidumbres relacionadas con la naturaleza estocástica de los procesos atmosféricos, son aspectos que se deben tener en cuenta para minimizar dichas diferencias adecuada concordante con los resultados del trabajo.

\section{AGRADECIMIENTOS}

Se agradece a la Ingeniera Ana Zuleima Orrego, supervisora de los convenios del Área Metropolitana del Valle de Aburrá (Convenio de Asociación No. 335 de 2016, Convenio de Asociación No. 315 de 2014) por las sugerencias en los documentos asociados a los resultados de los modelos CAMx y BRAMS, a la Profesora Miriam Gomez del Politécnico Colombiano Jaime Isaza Cadavid por los datos de las campañas de BC y a María Victoria Toro G. por la dirección de la tesis de Especialización de la UPB.

El artículo fue financiado por la Entidad o autoridad ambiental Área Metropolitana del Valle de Aburrá, a través de los convenios de asociación 315 de 2014 y 335 de 2016, con el Politécnico Colombiano Jaime Isaza Cadavid y la Universidad Pontificia Bolivariana. 


\section{CONFLICTOS DE INTERÉS DE LOS AUTORES}

Los autores declaran que no se presentan conflictos de interés.

\section{CONTRIBUCIÓN DE LOS AUTORES}

Juan Camilo Roldán-Vargas: Conceptualización, revisión, validación, análisis formal del trabajo, recursos, realización de los ensayos, escritura, adquisición de datos, conformación del marco teórico, análisis estadístico, realización de los ensayos.

María Victoria Toro-Gómez: Conceptualización, revisión, supervisión, escritura.

Alejandro Marín-Sánchez: Revisión.

\section{REFERENCIAS}

[1] Área Metropolitana del Valle de Aburrá; Universidad Pontificia Bolivariana, "Simulaciones Especiales. Tarea 1: actualización del inventario de emisiones atmosféricas," Convenio de Asociación No. 543 de 2008. p. 39, 2008. URL

[2] T. Ahmed; V. A. Dutkiewicz; A. J. Khan; L. Husain, "Long term trends in Black Carbon Concentrations in the Northeastern United States," Atmos. Res., vol. 137, pp. 49-57, Feb. 2014. https://doi.org/10.1016/j.atmosres.2013.10.003

[3] D. Koch et al., "Evaluation of black carbon estimations in global aerosol models," Atmos. Chem. Phys., vol. 9, no. 22, pp. 79-81, Nov. 2009. https://doi.org/10.5194/acp-9-9001-2009

[4] M. Ruppel; M. T. Lund; H. Grythe; N. L. Rose; J. Weckström; A. Korhola, "Comparison of spheroidal carbonaceous particle data with modelled atmospheric black carbon concentration and deposition and air mass sources in Northern Europe, 1850-2010," Adv. Meteorol., vol. 2013, Sep. 2013. https://doi.org/10.1155/2013/393926

[5] K. L. Denman et al., "Couplings Between Changes in the Climate System and Biogeochemistry" in Climate Change 2007: The Physical Science Basis. Contribution of Working Group I to the Fourth Assessment Report of the Intergovernmental Panel on Climate Change," Ed. Cambridge, United Kingdom and New York, NY, USA, 2007, pp. 2469-2479. URL

[6] E. J. Highwood; R. P. Kinnersley, "When smoke gets in our eyes: The multiple impacts of atmospheric black carbon on climate, air quality and health," Environ. Int., vol. 32, no. 4, pp. 560-566, May. 2006. https://doi.org/10.1016/j.envint.2005.12.003

[7] T. C. Bond et al., "Bounding the role of black carbon in the climate system: A scientific assessment," J. Geophys. Res. Atmos., vol. 118, no. 11, pp. 5380-5552, Jan. 2013. https://doi.org/10.1002/jgrd.50171

[8] W. W. Song; K. B. He; Y. Lei, "Black carbon emissions from on-road vehicles in China, 1990-2030," Atmos. Environ., vol. 51, pp. 320-328, May. 2012. https://doi.org/10.1016/j.atmosenv.2011.10.036

[9] D. T. Silverman et al., "The diesel exhaust in miners study: A nested case-control study of lung cancer and diesel exhaust," J. Natl. Cancer Inst., vol. 104, no. 11, pp. 855-868, Jun. 2012. https://doi.org/10.1093/jnci/djs034

[10] T. Novakov et al., "Large historical changes of fossil-fuel black carbon aerosols," Geophys. Res. Lett., vol. 30, no. 6, pp. 1-4, Mar. 2003. https://doi.org/10.1029/2002GL016345

[11] Área Metropolitana del Valle de Aburrá; Universidad Pontificia Bolivariana, "Actualización Inventario de emisiones del Valle de Aburrá, año 2015," Convenio de Asociación No. 335 de 2016. p. 86, Apr. 2018. URL

[12] Ramboll US Corporation, User's Guide Comprehensive air quality model with extensions version 6.50. Ramboll Environment and Health, 2018. URL

[13] Área Metropolitana del Valle de Aburrá; Universidad Pontificia Bolivariana, "Sistema de Modelización Atmosférica -SMA-," Convenio de Asociación No. 315 de 2014. p. 116, 2015. URL

[14] N. Davis; J. Lents; M. Osses; N. Nikkila; M. Barth, "Development and Application of an International Vehicle Emissions Model," Transp. Res. Rec., vol. 1939, no. 1, pp. 156-165, Jan. 2005. https://doi.org/10.1177/0361198105193900118

[15] R. Wang, Global emission inventory and atmospheric transport of black carbon: evaluation of the associated exposure. Springer-Verlag Berlin Heidelberg. 2015. https://doi.org/10.1007/978-3-662-46479-3

[16] Área Metropolitana del Valle de Aburrá; Universidad Pontificia Bolivariana, "Evaluación del 
funcionamiento del modelo meteorológico RAMs y del modelo de reacción química CAMx," Convenio de Asociación No. 075 de 2008. p. 42, 2017. URL

[17] C. Milford et al., "Measurements and simulation of speciated PM2.5 in south-west Europe," Atmos. Environ., vol. 77, 36-50, Oct 2013. https://doi.org/10.1016/j.atmosenv.2013.04.050 